\title{
Early responders within seven days of dupilumab treatment for severe asthma evaluated by patient-reported outcome: a pilot study
}

\author{
Nozomi Tani, Nobutaka Kataoka, Yusuke Kunimatsu, Yusuke Tachibana, Takumi Sugimoto, Izumi Sato, \\ Yuri Ogura, Kazuki Hirose, Takayuki Takeda
}

Department of Respiratory Medicine, Japanese Red Cross Kyoto Daini Hospital, Kyoto, Japan

Background: The management of severe asthma-associated symptoms is essential since they are distressing to the affected patients, and also greatly impair their quality of life. Dupilumab, a monoclonal antibody, blocks interleukin (IL)-4 and IL-13 signaling, both of which are crucial in acquired and innate immunity pathways through fast signal transduction, leading to an early response to treatment. Although rapid improvement within 1-3 days after dupilumab treatment was observed in moderate-to-severe atopic dermatitis, an early response within 7 days of dupilumab treatment in severe asthma has not been reported.

Methods: Twelve consecutive patients with severe asthma who were newly treated with dupilumab between July 2019 and April 2020 were retrospectively investigated. We evaluated the early response (within 7 days) of patients with severe asthma receiving dupilumab therapy. Asthma control test (ACT) and the daily ACT, which was modified from the ACT to evaluate daily symptoms associated with asthma, were adopted as patient-reported outcomes (PROs) at week 8 and within 7 days, respectively. Patients were stratified into early responders (7 days), late responders (week 8), and non-responders without significant improvement in PROs. Descriptive statistics were adopted due to the limited number of patients.

Results: Four of these 12 patients were early responders, with the following baseline characteristics: body mass index, $<25 \mathrm{~kg} / \mathrm{m}^{2}$; without depression; baseline forced expiratory volume in 1 second, $<1.50 \mathrm{~L}$; and more than one exacerbation in 1 year. On the other hand, five were late responders, and $44.4 \%$ of the nine responders were early responders. The higher the eosinophilic count and/or FeNO did not show any relationship between the early responder and nonresponder.

Conclusions: The effect of dupilumab on severe asthma in patients with atopic features could be started earlier than 2 weeks, similar to atopic dermatitis. Daily ACT may be useful in monitoring the early efficacy of dupilumab in treating severe asthma.

Key words: Asthma; daily ACT (asthma control test); dupilumab; early responder; severe asthma; quality of life.

Correspondence: Takayuki Takeda, Department of Respiratory Medicine, Japanese Red Cross Kyoto Daini Hospital, 355-5, Haruobi-cho, Kamanza-dori, Marutamachi-agaru, Kamigyo-ku, Kyoto 602-8026, Japan.

Tel.+81.75.2315171 - Fax:+81.75.2563451. E-mail: dyckw344@yahoo.co.jp

Contributions: NT, TT, designed and wrote the original manuscript; NK, YK, collected data; YT, TS, constructed methodology; IS, NT, analyzed the data; YO, KH, contributed to the final data curation and visualization; TT, supervised the study, reviewed and edited the manuscript. Each author made a significant contribution to the manuscript, has read and approved the final version of the manuscript and agreed to be accountable for all aspects of the work.

Conflict of interest: The authors declare that they have no competing interests, and all authors confirm accuracy.

Funding: None.

Availability of data and materials: The datasets analyzed in the current study are available from the corresponding author on reasonable request.

Ethics approval and consent to participate: This study was approved by the Ethics Committee of the Japanese Red Cross Kyoto Daini Hospital (approval date: 21 June 2019; approval number: S2019-21). The requirement for written informed consent was waived because of the retrospective study design and because patient anonymity was assured. Instead, the opportunity to opt-out was provided on the homepage of our hospital.

Consent for publication: Not applicable. 


\section{Introduction}

Asthma is one of the most common respiratory diseases characterized by heterogeneity in symptoms, severity, phenotypes, and endotypes due to the underlying molecular mechanisms [1]. In particular, severe asthma accounts for approximately 5 to $10 \%$ of the asthmatic population, and its management is of the utmost importance $[2,3]$. Severe asthma not only impairs the patient's quality of life (QOL), which can be life-threatening $[3,4]$, but also poses a high burden for both healthcare resources and society [3,5-7]. Thus, a comprehensive approach to effectively manage this condition is crucial.

To achieve this, the investigation into the molecular mechanism of airway inflammation has provided much-needed insight into the pathophysiology of severe asthma [8-10]. Both adaptive and innate immune systems are important in the pathogenesis of airway epithelial inflammation in asthma and play a pivotal role in type 2 inflammation. Adaptive immunity is mediated by T helper-2 (Th2) cells, eosinophils, immunoglobulin E (IgE)-producing B cells, and mast cells via IgE and Th2 cytokines such as interleukin-4 (IL-4), IL-5, and IL-13. On the other hand, group 2 innate lymphoid cells (ILC2s) play an important role via IL-5 and IL-13 in innate immunity. While upstream events in the airway epithelium, which trigger the production of $\mathrm{Th} 2$ cytokines, have been identified as thymic stromal lymphopoietin (TSLP) in adaptive immunity, and TSLP, IL-25, and IL-33 in innate immunity, respectively, biological agents targeting them have not been established in phase 3 trials.

The introduction of biological agents such as monoclonal antibodies targeting IgE (omalizumab), IL-5 (mepolizumab), and IL-5 receptor- $\alpha$ subunit (IL-5R $\alpha$; benralizumab) have revolutionized the treatment strategy for severe asthma [11-13]. They were applied according to the serum levels of IgE and eosinophil counts. Dupilumab is a monoclonal antibody that targets the anti-IL-4 receptor- $\alpha$ subunit (IL-4R $\alpha$ ) of the IL-4 and IL-4 / IL-13 receptor complexes, which blocks both IL-4 and IL-13 signaling. This is crucial in both acquired and innate immunity pathways [14].

Most of the primary endpoints in clinical trials of biological agents are established to confirm their long-term efficacy by setting endpoints at forced expiratory volume in 1 second $\left(\mathrm{FEV}_{1}\right)$, the annualized rate of severe asthma exacerbation, and the percentage reduction in the preexisting glucocorticoid dose more than 3 months after the treatment. Although these endpoints are significant in measuring the durable efficacy of biological agents, the early management of asthma-associated symptoms is equally important. Thus, the palliation of severe asthma-associated symptoms and an earlier response to biological agents is highly desired.

Although rapid improvement within 1-3 days after dupilumab treatment was observed in moderate-to-severe atopic dermatitis $[15,16]$, an early response within 7 days of dupilumab treatment in severe asthma has not been reported. We have modified the widely utilized asthma control test (ACT), a patient-reported outcome (PRO) [17] consisting of 5 items experienced within 4-weeks before assessing the frequency of dyspnea, general asthma-related symptoms, use of rescue medications, the effect of asthma on daily functioning, and overall self-assessment of asthma control [18]. Moreover, ACT is simple and useful in usual care settings and has been validated to be concordant with the Asthma Control Questionnaire (ACQ) [18]. ACT is more prevalent than ACQ in Japan. The minimal clinically important difference in ACT score over time is 3 points [19]. Since ACT was designed to report asthma control with a 4-week recall, it is unfit for the daily assessment of asthma-associated symptoms. Therefore, in the current study, ACT was modified for daily usage to report asthma control with a 24-hour recall, named "daily ACT," which could reflect daily symptoms and activity very well. Daily ACT was reported every day to evaluate the early response within 7 days of dupilumab treatment in severe asthma. The original ACT was also used to assess asthma-associated symptoms and the effect of dupilumab on 4-week asthma control. The baseline characteristics were also investigated in relation to the early responders within 7 days as well as responders at week 8 , who showed 3 points or more improvement in the daily ACT and original ACT scores, respectively.

\section{Methods}

\section{Patients}

A retrospective study was performed on 12 consecutive patients with severe asthma who were newly treated with dupilumab between July 2019 and April 2020.

These patients were asked to report both daily ACT and original ACT, which were routinely conducted at our hospital when biological agents were newly introduced irrespective of clinical study. Therefore, this study was categorized as retrospective. Those patients who had been treated with other biological agents and switched to dupilumab due to their ineffectiveness were not excluded irrespective of biological agent-free interval. This study was carried out in accordance with the Declaration of Helsinki and was approved by the Ethics Committee of the Japanese Red Cross Kyoto Daini Hospital (approval date: 21 June 2019; approval number: S2019-21). The requirement for written informed consent was waived because of the retrospective study design and also because patient anonymity was assured. Instead, the opportunity to opt-out was provided on the homepage of our hospital.

Dupilumab was introduced in patients with severe asthma who were uncontrolled under medium-to-high dose of inhaled corticosteroids (ICSs) and long-acting $\beta_{2}$-agonist (LABA) and/or long-acting muscarinic antagonist (LAMA). The indication criteria for dupilumab at our hospital were as follows: omalizumab was first introduced in patients who exhibited an allergic or atopic phenotype with elevated specific IgE antibodies and serum total IgE values ranging between 30 and $1500 \mathrm{IU} / \mathrm{mL}$, and switched to dupilumab when omalizumab was not effective; mepolizumab or benralizumab were introduced to patients who exhibited eosinophil counts of 150 cells $/ \mu \mathrm{L}$ or higher irrespective of an allergic or atopic phenotype, and switched to dupilumab when anti-IL$5 / \mathrm{IL}-5 \mathrm{R} \alpha$ therapies were ineffective; and dupilumab was preferentially introduced as the first-line biological agent to patients who demonstrated type 2 inflammation with elevated eosinophil counts or fraction of exhaled nitric oxide (FeNO) $\geq 25 \mathrm{ppb}$, when accompanied by atopic dermatitis or eosinophilic chronic rhinosinusitis.

\section{Treatment schedule, baseline characteristics, and evalu- ation of patient-related outcomes}

Dupilumab was administered subcutaneously at a dose of 600 $\mathrm{mg}$ on day 1 , followed by a dose of $300 \mathrm{mg}$ every 2 weeks as an add-on therapy.

The following baseline characteristics were analyzed: sex, age, body mass index (BMI), smoking habits, antigen-specific IgE, the highest serum total IgE level, and eosinophil counts $(\geq 300$ cells $/ \mu \mathrm{L}$ were evaluated as elevated) [20], FeNO ( $\geq 25$ ppb was evaluated as elevated) [21]; $\mathrm{FEV}_{1}$; past history of exacerbation requiring an emergency or urgent medical care visit and systemic corticosteroid treatment within 1 year before dupilumab introduction, prescription of leukotriene receptor antagonists (LTRAs), proton pump inhibitors (PPIs), macrolides, or oral corticosteroids (OCS), previous use of biological agents, previous bronchial thermoplasty (BT), and comorbidities including chronic obstructive 
Table 1. Daily asthma control test.

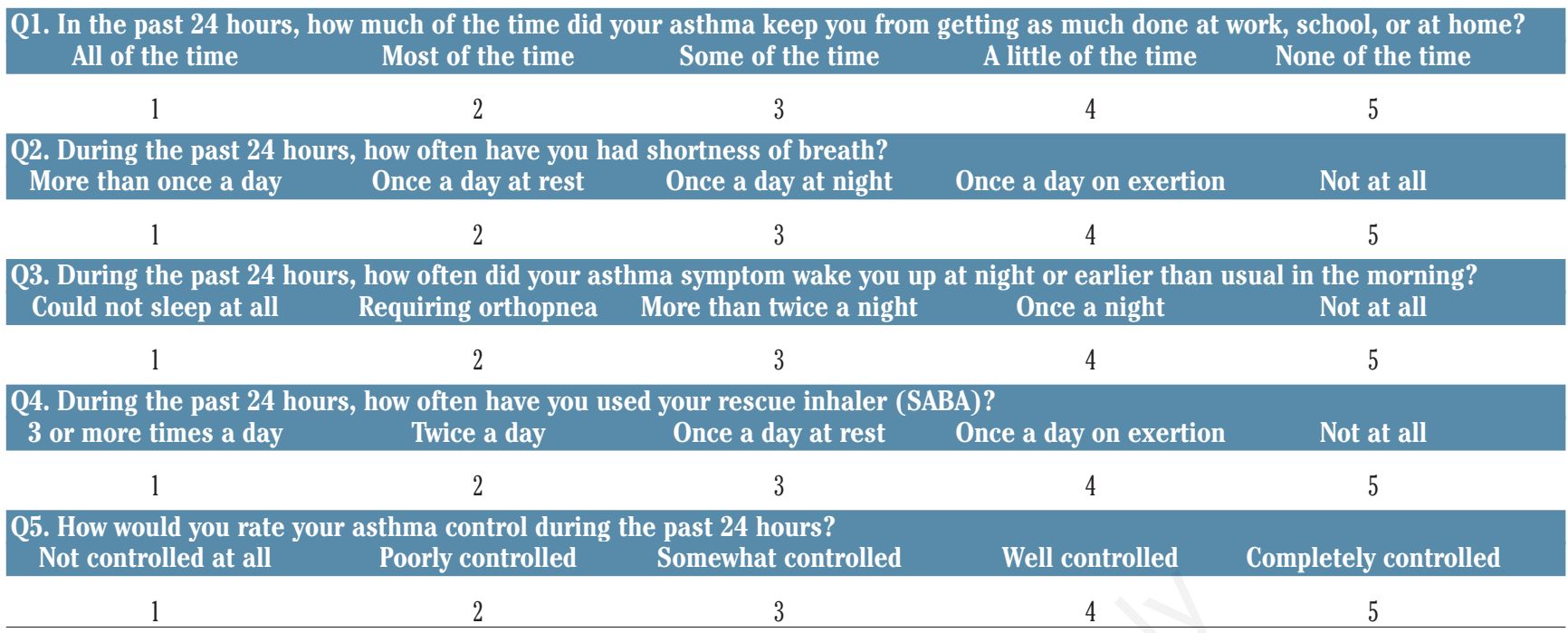

pulmonary disease (COPD), allergic rhinitis, eosinophilic chronic rhinosinusitis, atopic dermatitis, and depression.

Daily ACT was created as a 5-item, patient-administered survey evaluating the 24-hour control of asthma-associated symptoms. Asthma control with a 24 -hour recall was evaluated by 5 questions in accordance with the original ACT, while some modifications were made to questions No. 2, 3, and 4, which dealt with the frequency of asthma-associated symptoms and rescue inhaler use (Table 1). Daily ACT was reported by the patient every day for 8 successive days starting on the day of dupilumab introduction. The early response to dupilumab was defined as the improvement of 3 points or more in the daily ACT scores within 7 days after dupilumab introduction, where the daily ACT score on day 1 was used as the baseline in the evaluation. The definition of the early response as " 3 points or more improvements" was adopted through the interpretation of the original ACT [19]. The response to dupilumab was also defined as 3 points or more improvement in the original ACT scores at week 8, where the ACT score on day 1 was used as the baseline. The baseline scores were interchanged between the daily and the original ACT scores, since the contents of the daily ACT were modified from the original ACT, resulting in slightly different baseline scores between the two PROs.

\section{Statistical analysis}

Descriptive statistics were adopted to report the baseline characteristics, the evaluation of PROs, and the prevalence of early responders among the 12 patients in the 7 days period and the responder to dupilumab at week 8 because of the limited number of patients enrolled. The data are presented as median (range or standard deviation; SD) for continuous variables and percentages for categorical variables.

\section{Results}

\section{Baseline characteristics}

The baseline characteristics are summarized in Table 2. All the patients exhibited one or more factors associated with type 2 inflammation, that is, positive for antigen-specific $\operatorname{IgE}$, elevated eosinophil counts, or elevated FeNO levels at baseline. The median
ACT and the daily ACT scores at the baseline were 16 (range: 819) and 17 (range: 9-21), respectively. The median ACT and the daily ACT scores were relatively high since biological agents had been administered previously and were switched to dupilumab in 8

Table 2. Baseline characteristics. Total ( $\mathrm{n}=12)$

\begin{tabular}{lc} 
Age (median; years, range) & $51.5(38-77)$ \\
Sex (women / men; n, \%) & $10(83.3 \%) / 2(16.7 \%)$ \\
\hline BMI (median; kg/m², range) & $24.1(20.4-41.6)$ \\
Smoking habit + / - (n, \%) & $6(50.0 \%) / 6(50.0 \%)$ \\
\hline Antigen-specific IgE + / - (n, \%) & $12(100 \%) / 0(0 \%)$ \\
Total IgE (median; IU/mL, range) & $470(37-2470)$ \\
\hline Eosinophil counts (median; cells/ L, range) & $286.0(203.0-1368.0)$ \\
FeNO (median; ppb, range) & $41(7-300)$ \\
\hline FEV (median; ml, range) & $2070(500-3220)$ \\
ACT score at baseline (median; range) & $16(8-19)$ \\
\hline Daily ACT score at baseline (median; range) & $17(9-21)$ \\
More than one exacerbation + / - (n, \%) & $7(58.3 \%) / 5(41.7 \%)$ \\
\hline High-dose ICS/LABA + / - (n, \%) & $12(100 \%) / 0(0 \%)$ \\
LAMA + / - (n, \%) & $10(83.3 \%) / 2(16.7 \%)$ \\
\hline LTRA + / - (n, \%) & $11(91.7 \%) / 1(8.3 \%)$ \\
PPI + / - (n, \%) & $6(50.0 \%) / 6(50.0 \%)$ \\
\hline Macrolide + / - (n, \%) & $3(25.0 \%) / 9(75.0 \%)$ \\
Daily OCS + / - (n, \%) & $7(58.3 \%) / 5(41.7 \%)$ \\
\hline Biologic agents + / - (n, \%) & $8(66.7 \%) / 4(33.3 \%)$ \\
BT + / - (n, \%) & $2(16.7 \%) / 10(83.3 \%)$ \\
\hline COPD + / - (n, \%) & $3(25.0 \%) / 9(75.0 \%)$ \\
AR + / - (n, \%) & $2(16.7 \%) / 10(83.3 \%)$ \\
\hline ECRS + / - (n, \%) & $2(16.7 \%) / 10(83.3 \%)$ \\
AD + / - (n, \%) & $1(8.3 \%) / 11(91.7 \%)$ \\
\hline Depression + / - (n, \%) & $3(25.0 \%) / 9(75.0 \%)$ \\
\hline
\end{tabular}


patients, which are described below.

At baseline, high-dose ICS/LABA was prescribed for all the patients, LAMA for 10 (83.3\%), LTRAs for 11 (91.7\%), PPIs for 6 (50.0\%), macrolides for 3 (25.0\%), daily OCS for 7 (58.3\%), and biological agents had been previously administered in $8(66.7 \%$; omalizumab and benralizumab in 5 and 3 patients, respectively). The previous biological agents were directly switched to dupilum$\mathrm{ab}$ due to their inadequate effects in all 8 patients. In 2 patients, $(16.7 \%)$ BT was performed. The comorbidities observed were COPD in $3(25.0 \%)$, allergic rhinitis in $2(16.7 \%)$, eosinophilic chronic rhinosinusitis in $2(16.7 \%)$, atopic dermatitis in $1(8.3 \%)$, and depression in $3(25.0 \%)$ patients.

Changes in subjective symptoms of 12 patients via daily ACT within 7 days and the original ACT at weeks 4 and 8

The changes in PROs from the baseline of 12 patients via the daily ACT within 7 days (days 1-8) and the original ACT at weeks 4 and 8 are plotted in Figure 1. The median days needed for the significant improvement in the daily ACT in the 4 early responders was 5 days (range: $2-7$ days), while a significant improvement in the original ACT was observed for the first time in 7 patients at week 4 and in 2 patients at week 8 (Figure 1).

The patients were divided into 3 groups according to the response pattern: early responders within 7 days via the daily ACT, late responders until week 8 , and non-responders without significant improvement in PROs. The mean changes in the daily and the original ACT from the baseline (day 1) of these subpopulations are plotted in Figure 2. The fluctuation of the daily or original ACT scores was observed in some cases, while the scores tended to improve over time in both the early and late responders to dupilumab.

\section{Baseline characteristics according to the therapeutic effects of dupilumab on subjective symptoms within 7} days

Four out of 12 patients (33.3\%) reported an early response to dupilumab within 7 days, while the percentage of 4 early responders increased to $44.4 \%$ among 9 responders until week 8 . Three women and 1 man showed an early response, and all 4 early responders had a BMI $<25$ and without psychiatric disorders, while the median eosinophil counts and FeNO in these three patients were 391.9 (range: 203.0-1368.0) and 52 (range: 20-117), respectively. Three out of 4 early responders had baseline $\mathrm{FEV}_{1}$ less than $1.50 \mathrm{~L}$, and all 4 experienced more than 1 exacerbation within 1 year. On the other hand, the median eosinophil counts and FeNO in 8 patients without an early response were 286.0 (range, 211.2 593.6) and 30.5 (range, $7-300$ ), respectively. Six out of 8 patients without an early response had a baseline $\mathrm{FEV}_{1}>1.50 \mathrm{~L}$, and only 3 patients experienced more than 1 exacerbation within 1 year. There was no relationship between a higher eosinophilic count and/or FeNO with early and non-responder to dupilumab via the daily ACT within 7 days in the current study, due in part to a small sample size (Table 3).

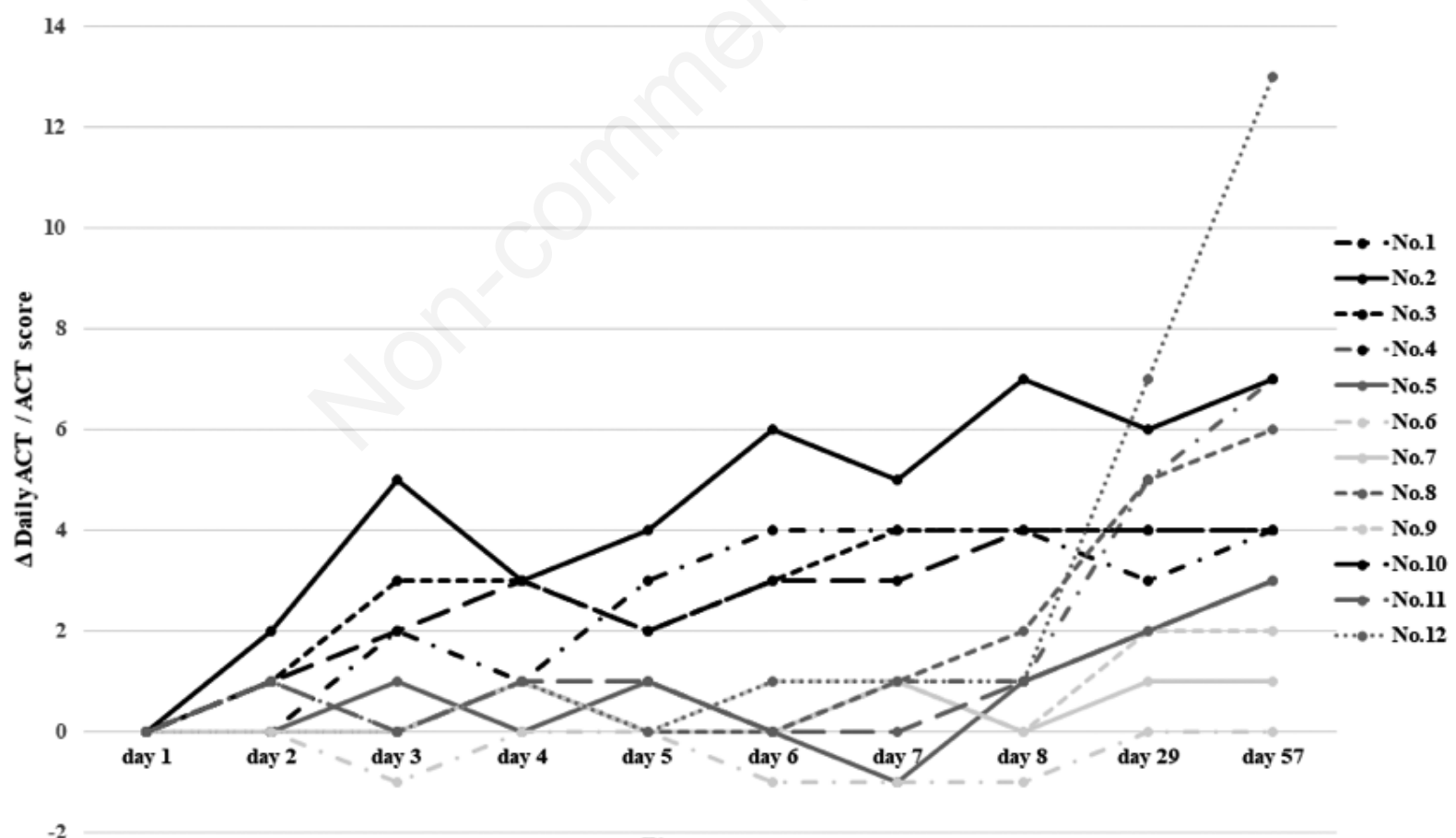

$-2$

Time course

Figure 1. The changes in patient-reported outcomes from the baseline of 12 patients via the daily asthma control test (ACT) on days 1-8 and the original ACT at weeks 4 and 8 are plotted. The black lines (No. 1, 2, 3, 10) represent the early responders within 7 days, the dark gray lines (No. 4, 5, 8, 11, and 12) the late responder until week 8, and the light gray lines (No. 6, 7, and 9) the non-responders, respectively. The median day needed for the significant improvement in 4 early responders was 5 days (range, 2-7 days), while a significant improvement in the early and late responders via the original ACT was observed for the first time in 7 patients at week 4 and in 2 patients at week 8 . 
Baseline characteristics according to the therapeutic effects of dupilumab on subjective symptoms at week 8

Nine out of 12 patients $(75.0 \%)$ responded to dupilumab via the original ACT until week 8, including 4 early responders within 7 days. The median eosinophil counts and FeNO in 9 responders were 287.0 (range, 203.0-1368.0) and 47 (range, 7-300), respectively. Although eosinophil counts and FeNO represent a type 2 inflammation, the higher eosinophilic count and/or FeNO did not show any relationship between responders and non-responders to dupilumab until week 8 in the current study because of the small sample size (Table 4). Three out of 9 responders had baseline FEV $<1.50 \mathrm{~L}$, and 6 out of 9 experienced more than 1 exacerbation within 1 year. All 3 non-responders were women, and had characteristics of BMI $\geq 28$ and comorbidity of depression, while the median eosinophil counts and FeNO in these 3 patients were 285 (range:

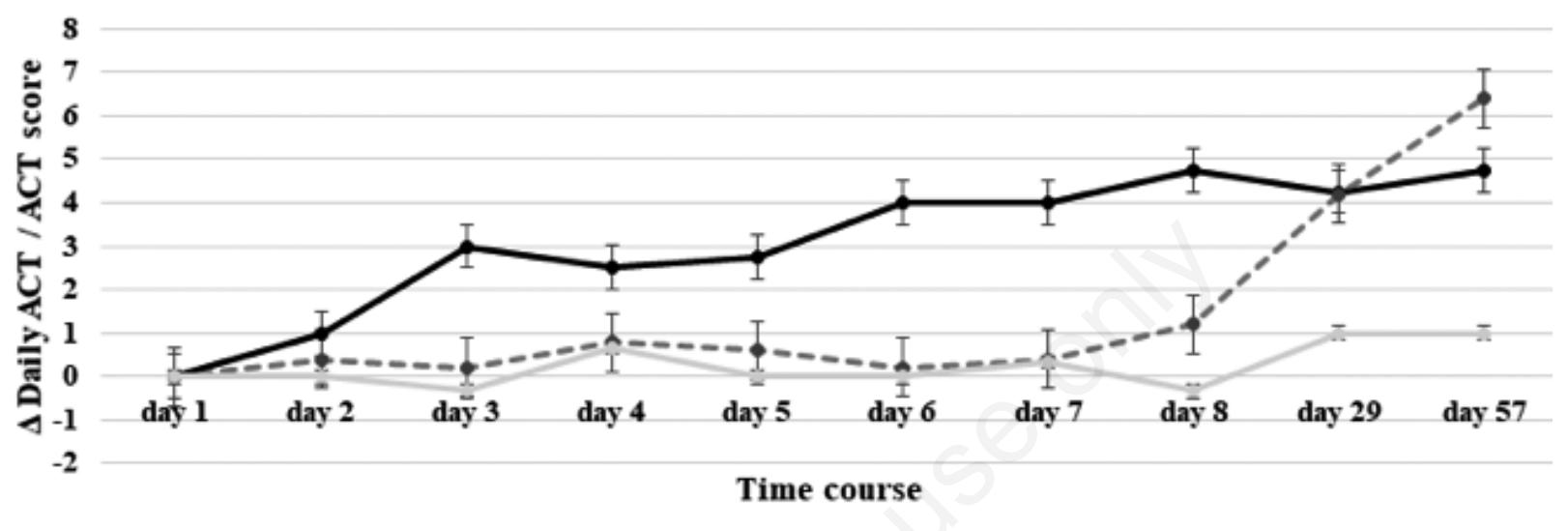

$\longrightarrow$ Early responders within 7 days $\quad \bullet-$ Late responders at week $8 \longrightarrow$ Non-responders at week 8

Figure 2. The mean changes in the daily and the original asthma control test (ACT) of the early responders within 7 days, the late responders until week 8 , and the non-responders were plotted. The early responders quickly reached the significant improvement in 2 days followed by further improvement. On the other hand, the late responders showed significant improvement at week 4 followed by further improvement until week 8. The error bars show an SD of 2.

Table 3. Baseline characteristics according to the therapeutic effects of dupilumab on subjective symptoms within 7 days.

\begin{tabular}{|c|c|c|c|}
\hline & Total $(n=12)$ & Early responders $(n=4)$ & Late or non-responders $(\mathrm{n}=8)$ \\
\hline Age (median; years, range) & $51.5(38-77)$ & $70.0(38-75)$ & $49.5(41-77)$ \\
\hline Sex (women / men; n, \%) & $10(83.3 \%) / 2(16.7 \%)$ & $3(75.0 \%) / 1(25.0 \%)$ & $7(87.5 \%) / 1(12.5 \%)$ \\
\hline BMI (median; kg/m², range) & $24.1(20.4-41.6)$ & $21.9(20.4-24.8)$ & $26.7(20.7-41.6)$ \\
\hline Smoking habit $+/-(n, \%)$ & $6(50.0 \%) / 6(50.0 \%)$ & $1(25.0 \%) / 3(75.0 \%)$ & $5(62.5 \%) / 3(37.5 \%)$ \\
\hline Total IgE (median; IU/mL, range) & $470(37-2470)$ & $407(267-2470)$ & $472.5(37-1791)$ \\
\hline Eosinophil counts (median; cells/ L, range) & $286.0(203.0-1368.0)$ & $391.9(203.0-1368.0)$ & $286.0(211.2-593.6)$ \\
\hline FeNO (median; ppb, range) & $41.0(7-300)$ & $52.0(20-117)$ & $30.5(7-300)$ \\
\hline $\mathrm{FEV}_{1}$ (median; ml, range) & $2070(500-3220)$ & $1415(500-3220)$ & $2370(1240-2970)$ \\
\hline ACT score at baseline (median; range) & $16(8-19)$ & $15(13-17)$ & $16(8-19)$ \\
\hline Daily ACT score at baseline (median; range) & $17(9-21)$ & $15(13-17)$ & $17(9-21)$ \\
\hline More than one exacerbation + / - $(\mathrm{n}, \%)$ & $7(58.3 \%) / 5(41.7 \%)$ & $4(100 \%) / 0(0 \%)$ & $3(37.5 \%) / 5(62.5 \%)$ \\
\hline Macrolide + / - (n, \%) & $3(25.0 \%) / 9(75.0 \%)$ & $2(50.0 \%) / 2(50.0 \%)$ & $1(12.5 \%) / 7(87.5 \%)$ \\
\hline Daily OCS + / - (n, \%) & $7(58.3 \%) / 5(41.7 \%)$ & $3(75.0 \%) / 1(25.0 \%)$ & $4(50.0 \%) / 4(50.0 \%)$ \\
\hline Biologic agents + / - (n, \%) & $8(66.7 \%) / 4(33.3 \%)$ & $3(75.0 \%) / 1(25.0 \%)$ & $5(62.5 \%) / 3(37.5 \%)$ \\
\hline COPD + / - (n, \%) & $3(25.0 \%) / 9(75.0 \%)$ & $1(25.0 \%) / 3(75.0 \%)$ & $2(25.0 \%) / 6(75.0 \%)$ \\
\hline $\mathrm{AR}+/-(\mathrm{n}, \%)$ & $2(16.7 \%) / 10(83.3 \%)$ & $0(0 \%) / 4(100 \%)$ & $2(25.0 \%) / 6(75.0 \%)$ \\
\hline $\mathrm{ECRS}+/-(\mathrm{n}, \%)$ & $2(16.7 \%) / 10(83.3 \%)$ & $0(0 \%) / 4(100 \%)$ & $2(25.0 \%) / 6(75.0 \%)$ \\
\hline$A D+/-(n, \%)$ & $1(8.3 \%) / 11(91.7 \%)$ & $0(0 \%) / 4(100 \%)$ & $1(12.5 \%) / 7(87.5 \%)$ \\
\hline Depression + / - (n, \%) & $3(25.0 \%) / 9(75.0 \%)$ & $0(0 \%) / 4(100 \%)$ & $3(37.5 \%) / 5(62.5 \%)$ \\
\hline
\end{tabular}

ACT, asthma control test; AD, atopic dermatitis; AR, allergic rhinitis; BMI, body mass index; BT, bronchial thermoplasty; ECRS, eosinophilic chronic rhinosinusitis; FeNO, a fraction of exhaled nitric oxide; $\mathrm{FEV}_{1}$, forced expiratory volume in 1 second; ICS, inhaled corticosteroid; IgE, immunoglobulin E; LABA, long-acting $\beta_{2}$-agonist; LAMA, long-acting anti-muscarinic antagonist; LTRA, leukotriene receptor antagonist; OCS, oral corticosteroid; PPI, proton pump inhibitors. 
211.2-378) and 23 (range: 16-38), respectively, without any difference compared to those of the responders. All 3 non-responders had baseline $\mathrm{FEV}_{1}>1.50 \mathrm{~L}$, and only 1 non-responder experienced more than 1 exacerbation within 1 year before dupilumab introduction.

\section{Discussion}

An appropriate assessment of asthma control is essential in clinical practice, which is difficult due to its heterogeneous nature [1]. Therefore, physicians' underestimation of asthma-associated symptoms [22], which can lead to the worsening of the patients' QOL, should be avoided. Incorporation of PROs, including ACT and lung function tests, is essential for appropriate asthma management, and avoiding patients'-physicians' discordance in the QOL assessment. Under such circumstances, physical activity, which is often hampered by asthma-associated symptoms, is very important in asthma control since it sometimes leads to worsening of asthma control [23]. Therefore, physical activity and QOL should be improved as soon as possible. Lung function including $\mathrm{FEV}_{1}$, asthma-associated symptoms, and QOL often diverges from one another [18]. Thus, the assessment of asthma control regularly through $\mathrm{PRO}$ as well as $\mathrm{FEV}_{1}$ remains equally important.

Dupilumab demonstrated efficacy in reducing the risk of severe asthma exacerbation and maintaining $\mathrm{FEV}_{1}$ in moderate-tosevere uncontrolled asthma, especially in patients with an eosinophil count of 300 or more per $\mathrm{mm}^{3}$ [24], and it also reduced oral glucocorticoid use in steroid-dependent severe asthma [25].

On the other hand, the efficacy of dupilumab in asthma-associated symptoms, QOL, and productivity in asthmatic patients has been evaluated at week 24 after dupilumab treatment using ACQ5 and asthma quality of life questionnaire (AQLQ), and dupilumab significantly improved ACQ-5 and AQLQ scores [26]. However, the early efficiency of dupilumab within 7 days has not been reported. This could be due to the lack of quantitative assessment of the QOL daily as well as the administration interval of dupilumab every 2 weeks.

In the current study, we evaluated the changes in asthma-associated QOL on a daily basis for 8 consecutive days, including the day of dupilumab introduction using the daily ACT to detect early responders to dupilumab. The original ACT was also used every 4 weeks, and the efficacy was evaluated at week 8 after treatment. Nine patients $(75.0 \%)$ showed efficacy via the original ACT at weeks 8 , and $4(44.4 \%)$ out of 9 responders achieved significant improvement in daily ACT score within 7 days after dupilumab treatment. Although descriptive statistics were adopted due to the limited number of patients, all 4 early responders to dupilumab had characteristics of BMI lower than 25 and were without psychiatric disorders. All 4 patients had experienced more than 1 exacerbation before dupilumab introduction and 3 out of 4 had a baseline FEV $<1.50 \mathrm{~L}$, which was in concordance with the observed results in a previous phase $2 \mathrm{~b}$ study investigating the efficacy of dupilumab on symptoms and QOL in patients with persistent asthma [26]. On the other hand, the higher the eosinophilic count and/or FeNO did not show any relationship between the early responder and nonresponder.

Dupilumab is an anti-IL-4R $\alpha$ antibody that inhibits both IL-4 and IL-13 signaling by inhibiting the IL-13 receptor- $\alpha 1$ subunit (IL-13R 1 1) [14]. IL-4 and IL-13 are the key Th2 cytokines, with IL-4 being secreted from Th2 cells and IL-13 from both Th2 cells and ILC2s. IL-4 plays an important role in polarization and functional maintenance of Th2 cells, while IL-13 induces the expression of inducible nitric oxide synthase (iNOS) in the airway epithelium as well as plays roles in TSLP promotion, goblet cell hyperplasia, and airway remodeling through extracellular collagen depo-

Table 4. Baseline characteristics according to the therapeutic effects of dupilumab on subjective symptoms at week 8.

\begin{tabular}{|c|c|c|c|}
\hline & Total $(n=12)$ & Responders $(\mathrm{n}=9)$ & Non-responders $(\mathrm{n}=3)$ \\
\hline Age (median; years, range) & $51.5(38-77)$ & $51.0(38-77)$ & $52.0(41-64)$ \\
\hline Sex (women / men; n, \%) & $10(83.3 \%) / 2(16.7 \%)$ & $7(77.8 \%) / 2(22.2 \%)$ & $3(100 \%) / 0(0 \%)$ \\
\hline BMI (median; kg/m², range) & $24.1(20.4-41.6)$ & $22.2(20.4-29.9)$ & $33.7(28.2-41.6)$ \\
\hline Smoking habit + / - (n, \%) & $6(50.0 \%) / 6(50.0 \%)$ & $4(44.4 \%) / 5(55.6 \%)$ & $2(75.0 \%) / 1(25.0 \%)$ \\
\hline Total IgE (median; IU/mL, range) & $470(37-2470)$ & $408(37-2470)$ & $537(280-829)$ \\
\hline Eosinophil counts (median; cells/ $\mu \mathrm{L}$, range) & $286.0(203.0-1368.0)$ & $287.0(203.0-1368.0)$ & $285.0(211.2-378.0)$ \\
\hline FeNO (median; ppb, range) & $41(7-300)$ & $47(7-300)$ & $23(16-38)$ \\
\hline $\mathrm{FEV}_{1}$ (median; ml, range) & $2070(500-3220)$ & $2390(500-3220)$ & $1390(1240-2350)$ \\
\hline ACT score at baseline (median; range) & $16(8-19)$ & $16(12-18)$ & $16(8-19)$ \\
\hline Daily ACT score at baseline (median; range) & $17(9-21)$ & $17(12-18)$ & $17(9-21)$ \\
\hline More than one exacerbation + / - (n, \%) & $7(58.3 \%) / 5(41.7 \%)$ & $6(66.7 \%) / 3(33.3 \%)$ & $1(33.3 \%) / 2(66.7 \%)$ \\
\hline Macrolide + / - (n, \%) & $3(25.0 \%) / 9(75.0 \%)$ & $2(22.2 \%) / 7(77.8 \%)$ & $1(33.3 \%) / 2(66.7 \%)$ \\
\hline Daily OCS + / - (n, \%) & $7(58.3 \%) / 5(41.7 \%)$ & $5(55.6 \%) / 4(44.4 \%)$ & $2(75.0 \%) / 1(25.0 \%)$ \\
\hline Biologic agents + / - (n, \%) & $8(66.7 \%) / 4(33.3 \%)$ & $6(66.7 \%) / 3(33.3 \%)$ & $2(75.0 \%) / 1(25.0 \%)$ \\
\hline COPD + / - (n, \%) & $3(25.0 \%) / 9(75.0 \%)$ & $2(22.2 \%) / 7$ (77.8\%) & $1(33.3 \%) / 2(66.7 \%)$ \\
\hline $\mathrm{AR}+/-(\mathrm{n}, \%)$ & $2(16.7 \%) / 10(83.3 \%)$ & $2(22.2 \%) / 7(77.8 \%)$ & $0(0 \%) / 3(100 \%)$ \\
\hline ECRS + / - (n, \%) & $2(16.7 \%) / 10(83.3 \%)$ & $2(22.2 \%) / 7(77.8 \%)$ & $0(0 \%) / 3(100 \%)$ \\
\hline $\mathrm{AD}+/-(\mathrm{n}, \%)$ & $1(8.3 \%) / 11(91.7 \%)$ & $1(11.1 \%) / 8(88.9 \%)$ & $0(0 \%) / 3(100 \%)$ \\
\hline Depression + / - (n, \%) & $3(25.0 \%) / 9(75.0 \%)$ & $0(0 \%) / 9(100 \%)$ & $3(100 \%) / 0(0 \%)$ \\
\hline
\end{tabular}

ACT, asthma control test; AD, atopic dermatitis; AR, allergic rhinitis; BMI, body mass index; BT, bronchial thermoplasty; ECRS, eosinophilic chronic rhinosinusitis; FeNO, a fraction of exhaled nitric oxide; $\mathrm{FEV}_{1}$, forced expiratory volume in 1 second; ICS, inhaled corticosteroid; IgE, immunoglobulin E; LABA, long-acting $\beta_{2}$-agonist; LAMA, long-acting anti-muscarinic antagonist; LTRA, leukotriene receptor antagonist; OCS, oral corticosteroid; PPI, proton pump inhibitors. 
sition $[14,27]$. These mechanisms are essential in airway hypersensitivity, which is a crucial part of the pathophysiology of severe asthma. Both IL-4R $\alpha$ and IL-13R $\alpha 1$ are expressed in B lymphocytes, eosinophils, antigen-presenting cells, and bronchial epithelial cells, and consist of a heterodimeric receptor complex [28]. The activation of the heterodimeric complex of IL-4R $\alpha$ and IL$13 \mathrm{R} \alpha 1$ induces prompt stimulation of Janus kinase $1 / 2$ (JAK 1/2) and tyrosine kinase 2 (Tyk2), followed by phosphorylation of signal transducer and activator transcription 6 (STAT6) [29,30]. Signal transduction through the cascade of JAK $1 / 2$, Tyk2, and following STAT6 is very quick; therefore, blocking IL-4 and/or IL-13 is considered very promising. Anti-IL-13 antibodies tralokinumab [31] and lebrikizumab [32] failed to demonstrate consistent clinical benefits in phase 3 trials. On the other hand, treatments that solely target IL-4 have never proven efficacy in severe asthma $[33,34]$. Under circumstances where the inhibition of either IL-4 or IL-13 signaling is not effective in severe asthma, dual inhibition of IL-4 and IL-13 with dupilumab demonstrated clinical benefit [24,25].

The early responders within 7 days after dupilumab treatment, consisting of $44.4 \%$ of all responders, could be explained by the basic mechanism mentioned earlier. The dual inhibition of IL-4 and IL-13 suppresses Th2 cell activation and iNOS induction, resulting in controlled airway hypersensitivity. These effects could be quickly noticed by patients considering prompt signal transduction through JAK $1 / 2$, Tyk2, and STAT6. The early efficacy of dupilumab in moderate-to-severe atopic dermatitis has been observed in the pooled analysis of LIVERTY AD SOLO 1 and 2 [15] within 1-3 days both subjective (by patient-reported itch) and objective (by eczema area severity index), and combined analysis of SOLO 1 and 2, AD ADOL, and CHRONOS by day 2 in adults and by day 5 in adolescents subjectively (daily peak pruritis numerical rating scale score) [16]. Therefore, the observed early responders to dupilumab treatment in severe asthma are concordant with both basic mechanisms and previously confirmed clinical effects on moderate-to-severe atopic dermatitis. In addition to the dual inhibiting effect of dupilumab, the loading dose of dupilumab on day $1(600 \mathrm{mg})$ could have exerted a very rapid effect on asthma-associated symptoms, allowing systemic concentrations to peak enough for strong suppression of IL-4 and IL-13 signals before reaching a steady state.

There are some limitations to the current study. First, since this was a retrospective observational study design with limited sample size and without controls (placebo), the bias may be higher compared to prospective studies with larger populations. Second, since the daily ACT is different from the original ACT, the definition of 3 points of improvement as the minimal clinically important difference over time in the daily ACT score has not been validated and needs validation through prospective studies. Nevertheless, its definition was adopted in accordance with the original ACT. The daily ACT was feasible in the current study, which was in concordance with the clinical course during the first 7 days, with all of the early responders assessed by the daily ACT, resulting in effective ACT at week 8 .

\section{Conclusions}

Taken together, the effect of dupilumab on severe asthma with atopic features was observed within 7 days in $44.4 \%$ of the patients who showed improvement in ACT scores after 8 weeks of treatment. Thus, the effect of dupilumab could have started earlier than 2 weeks in severe asthma. Daily ACT may be useful in monitoring the early efficacy of dupilumab in treating severe asthma.

\section{Abbreviations}

ACT, asthma control test;

ACQ, asthma control questionnaire;

AQLQ, asthma quality of life questionnaire;

BMI, body mass index;

BT, bronchial thermoplasty;

COPD, chronic obstructive pulmonary disease;

FeNO, fraction of exhaled nitric oxide;

$\mathrm{FEV}_{1}$, forced expiratory volume in 1 second;

ICS, inhaled corticosteroid;

Ig, immunoglobulin;

iNOS, inducible nitric oxide synthase;

IL, interleukin;

IL-4R $\alpha, \quad$ IL-4 receptor- $\alpha$ subunit;

IL-5R $\alpha, \quad$ IL-5 receptor- $\alpha$ subunit;

IL-13R $\alpha 1$, IL-13 receptor- $\alpha 1$ subunit; interleukin;

ILC2, group 2 innate lymphoid cell;

JAK $1 / 2$, Janus kinase $1 / 2$;

LABA, long-acting $\beta_{2}$-agonist;

LAMA, long-acting anti-muscarinic antagonist;

LTRA, leukotriene receptor antagonist;

OCS, oral corticosteroid;

PPI, $\quad$ proton pump inhibitor;

PRO, patient-reported outcome;

SD, standard deviation;

STAT6, signal transducer and activator transcription 6;

Th2, T helper-2;

TSLP, thymic stromal lymphopoietin;

Tyk2, tyrosine kinase 2;

QOL, quality of life.

\section{References}

1. Global Initiative for Asthma, Global Strategy for Asthma Management and Prevention. Updated 2020. Accessed on: 26 June 2020. Available from: https://ginasthma.org/wpcontent/uploads/2020/06/GINA-2020-report 20_06 04-1wms.pdf

2. Hekking PP, Wener RR, Amelink M, Zwinderman AH, Bouvy ML, Bel EH. The prevalence of severe refractory asthma. J Allergy Clin Immunol 2015;135:896-902.

3. Chipps BE, Haselkorn T, Paknis B, Ortiz B, Bleecker ER, Kianifard F, et al. Epidemiology and natural history of asthma: Outcomes and Treatment Regimens Study Group. More than a decade follow-up in patients with severe or difficult-to-treat asthma: The epidemiology and natural history of asthma: outcomes and treatment regimens (TENOR) II. J Allergy Clin Immunol 2018;141:1590-7.

4. Kupczyk M, ten Brinke A, Sterk PJ, Bel EH, Papi A, Chanez $\mathrm{P}$, et al. Frequent exacerbators--A distinct phenotype of severe asthma. Clin Exp Allergy 2014;44:212-21.

5. Mazalovic K, Jacoud F, Dima AL, Van Ganse E, Nolin MCD, Zaba C. The Astro-Lab Group. Asthma exacerbations and socio-economic status in French adults with persistent asthma: A prospective cohort study. J Asthma 2018;55:1043-51.

6. Van Ganse E, Antonicelli L, Zhang, Q, Laforest L, Yin DD, Nocea G, et al. Asthma-related resource use and cost by GINA classification of severity in three European countries. Respir Med 2006;100:140-7.

7. Sullivan PW, Ghushchyan VH, Globe G, Schatz M. Oral corticosteroid exposure and adverse effects in asthmatic patients. J 
Allergy Clin Immunol 2018;141:110-6.

8. Fahy JV. Type 2 inflammation in asthma - present in most, absent in many. Nat Rev Immunol 2015;15:57-65.

9. Hirose K, Iwata A, Tamachi T, Nakajima H. Allergic airway inflammation: key players beyond the Th2 cell pathway. Immunol Rev 2017;278:145-61.

10. Kuruvilla ME, Lee FE-H, Lee GB. Understanding asthma phenotypes, endotypes, and mechanism of disease. Clin Rev Allergy Immunol 2019;56:219-33.

11. D'Amato G, Stanziola A, Sanduzzi A, Liccardi G, Salzillo A, Vitale $\mathrm{C}$, et al. Treating severe allergic asthma with anti-IgE monoclonal antibody (omalizumab): a review. Multidiscip Respir Med 2014;9:23.

12. Menzella F, Lusuardi M, Galeone C, Zucchi L. Tailored therapy for severe asthma. Multidiscip Respir Med 2015;10:1.

13. Busse WW. Biological treatments for severe asthma: A major advance in asthma care. Allergol Int. 2019; 68: 158-66.

14. Barranco P, Phillips-Angles E, Dominguez-Ortega J, Quirce S. Dupilumab in the management of moderate-to-severe asthma: the data so far. Ther Clin Risk Manag 2017;13:1139-49.

15. Thaçi, D, L Simpson E, Deleuran M, Kataoka Y, Chen Z, Gadkari A, et al. Efficacy and safety of dupilumab monotherapy in adults with moderate-to-severe atopic dermatitis: A pooled analysis of two phase 3 randomized trials (LIBERTY AD SOLO 1 and LIBERTY AD SOLO 2). J Dermatol Sci 2019;94:266-75.

16. Silverberg JI, Yosipovitch G, Simpson EL, Kim BS, Wu JJ, Eckert L, et al. Dupilumab treatment results in early and sustained improvements in itch in adolescents and adults with moderate to severe atopic dermatitis: Analysis of the randomized phase 3 studies SOLO 1 and SOLO 2, AD ADOL, and CHRONOS. J Am Acad Dermatol 2020;82:1328-36.

17. Swigris JJ, Fairclough D. Patient-reported outcomes in idiopathic pulmonary fibrosis research. Chest 2012;142:291-7.

18. Schatz M, Sorkness CA, Li JT, Marcus P, Murray JJ, Nathan RA, et al. Asthma Control Test: Reliability, validity, and responsiveness in patients not previously followed by asthma specialists. J Allergy Clin Immunol 2006;117:549-56.

19. Schatz M, Kosinski M, Yarlas AS, Hanlon J, Watson ME, Jhingran P. The minimally important difference of the asthma control test. J Allergy Clin Immunol 2009;124:719-23.

20. Yancey SW, Keene ON, Albers FC, Ortega H, Bates S, Bleecker ER, et al. Biomarkers for severe eosinophilic asthma. J Allergy Clin Immunol 2017;140:1509-18.

21. Bjermer L, Alving K, Diamant Z, Magnussen H, Pavord I, Piacentini G, et al. Current evidence and future research needs for FeNO measurement in respiratory diseases. Respir Med 2014; 108 830-41.
22. Matsunaga K, Hamada K, Oishi K, Yano M, Yamaji Y, Hirano T. Factors associated with physician-patient discordance in the perception of asthma control. J Allergy Clin Immunol Pract 2019;7:2634-41.

23. Panagiotou M, Koulouris NG, Rovina N. Physical activity: A missing link in asthma care. J Clin Med 2020;9:706.

24. Castro M, Corren J, Pavord ID, Maspero J, Wenzel S, Rabe $\mathrm{KF}$, et al. Dupilumab efficacy and safety in moderate-to-severe uncontrolled asthma. N Engl J Med 2018;378:2486-96.

25. Rabe KF, Nair P, Brusselle G, Maspero JF, Castro M, Sher L, et al. Efficacy and safety of dupilumab in glucocorticoiddependent severe asthma. N Engl J Med 2018;378:2475-85.

26. Corren J, Castro M, Chanez P, Fabbri L, Joish VN, Amin N, et al. Dupilumab improves symptoms, quality of life, and productivity in uncontrolled persistent asthma. Ann Allergy Asthma Immunol 2019;122:41-9.

27. Barnes PJ. Targeting cytokines to treat asthma and chronic obstructive pulmonary disease. Nat Rev Immunol 2018;18:454-66.

28. LaPorte SL, Juo ZS, Vaclavikova J, Colf LA, Qi X, Heller NM, et al. Molecular and structural basis of cytokine receptor pleiotropy in the interleukin-4/13 system. Cell 2008;132:25972.

29. Oh CK, Geba GP, Molfino N. Investigational therapeutics targeting the IL-4/IL-13/STAT-6 pathway for the treatment of asthma. Eur Respir Rev 2010;19:46-54.

30. Chiba Y, Goto K, Misawa M. Interleukin-13-induced activation of signal transducer and activator of transcription 6 is mediated by activation of Janus kinase 1 in cultured human bronchial smooth muscle cells. Pharmacol Rep 2012;64:454-8.

31. Panettieri RA Jr, Sjöbring U, Péterffy A, Wessman P, Bowen K, Piper E, et al. Tralokinumab for severe, uncontrolled ssthma (STRATOS 1 and STRATOS 2): Two randomized, doubleblind, placebo-controlled, phase 3 clinical trials. Lancet Respir Med 2018;6:511-25.

32. Hanania NA, Korenblat P, Chapman KR, Bateman ED, Kopecky P, Paggiaro P, et al. Efficacy and safety of lebrikizumab in patients with uncontrolled asthma (LAVOLTA I and LAVOLTA II): Replicate, phase 3, randomized, double-blind, placebo-controlled trials. Lancet Respir Med 2016;4:781-96.

33. Wenzel S, Wilbraham D, Fuller R, Getz EB, Longphre M. Effect of an interleukin-4 variant on late-phase asthmatic response to allergen challenge in asthmatic patients: results of two phase 2a studies. Lancet 2007;370:1422-31.

34. Corren J, Busse W, Meltzer EO, Mansfield L, Bensch G, Fahrenholz J, et al. A randomized, controlled, phase 2 study of AMG 317, an IL-4Ralpha antagonist, in patients with asthma. Am J Respir Crit Care Med 2010;181:788-96. 